\title{
KEY AREAS OF ENGINEERING MECHANICS IN DESIGN OF MODERN INTEGRATED PROCESS EQUIPMENT
}

\author{
Jegla Z.*, Reppich M.**, Krňávek M.***, Horsák J.****
}

\begin{abstract}
Process engineering is a technical specialization focused on the design of industrial processes and equipment whose purpose is generally to convert raw materials into desired products. These include, for example, not only chemical or refinery processes, but also food and pharmaceutical processes, waste treatment processes and many others. Coverage of such a wide range of different technologies enables the concept of unit operations completed by a process integration technique allowing an efficient sequencing of unit operations in a process so that the process is designed and operated as cost-effectively as possible. In the context of global changes and the need for maximum energy efficient and environmentally friendly solutions of production processes, a new trend of so-called modern integrated equipment (MIE) is now starting. The MIE are generally characterized by maximum efficiency and multifunctionality via aggregation of multiple unit operations into a single apparatus. It reduces the process to the minimum number of equipment, thus reducing both investment and operating costs while process works efficiently and more environmentally. The MIE design principles are presented in the paper together with several design challenges addressed to the relevant key areas of engineering mechanics to which attention in the paper will be also paid.
\end{abstract}

\section{Keywords: Process Engineering, Process Integration, Modern Integrated Equipment, Design, Mechanics.}

\section{Introduction}

The technical specialization "Process Engineering" generally focuses on the design of industrial processes and equipment converting raw materials into desired products. This includes a very wide portfolio of technologically diverse production processes, including, for example: (i) crude oil and gas processing processes (i.e. refinery processes and petrochemical processes); (ii) processes of the chemical industry (production of washing powders, plastics, fertilizers, etc.); (iii) food industry processes (breweries and malt houses, sugar factories, dairies, chocolate factories, etc.); (iv) pharmaceutical and cosmetic industry processes; (v) consumer industry processes (such as ceramic, building material processing, rubber, paper and pulp production, etc.); (vi) biotechnological processes and processes for environmental protection (e.g. waste water treatment plants, processes for thermal and non-thermal waste disposal, units for purification of exhalations from process and power industries, etc.) and other production processes.

In order to effectively cover such a very wide range of different process technologies, process engineering uses the established concept of so-called "unit operations". By means of this concept, any production process is then viewed as a sequence of unit operations which in the process ensures the appropriate conversion of the raw material to the desired product. For example, the unit operation "heat exchange" is one of the fundamental unit operations as it is necessary for most production processes (e.g. in the production of gasoline from petroleum, fruit distillate from ferment, etc.). Each unit operation can then be performed in the process either by one equipment (apparatus) or by a group of equipment (i.e. several apparatuses).

\footnotetext{
* Assoc. Prof. Zdeněk Jegla, PhD.: Institute of Process Engineering, Faculty of Mechanical Engineering, Brno University of Technology; Technická 2896/2; 61669 Brno; CZ, zdenek.jegla@vut.cz

** Prof. Dr.-Ing. Marcus Reppich: Faculty of Mechanical and Process Engineering, Augsburg University of Applied Sciences; An der Hochschule 1; 86161 Augsburg; DE, marcus.reppich@hs-augsburg.de

*** Ing. Martin Krňávek: EVECO Brno, Březinova 42, 61600 Brno, CZ, krnavek@evecobrno.cz

**** Ing. Jan Horsák: UNIS, Jundrovská 33, 62400 Brno, CZ, jhorsak@unis.cz
} 
Process engineering uses process systems engineering methods and approaches for efficient design the sequence and interconnection of unit operations respectively process equipment that realize these unit operations. These process systems engineering techniques are collectively referred to as process integration, as presented, for example, by Klemeš et al. (2018). The main task of process integration is to effectively arrangement of the unit operations (and the equipment that implement them) so that the whole process is designed and operated as cost-effectively as possible.

Let us show on a simple process example (from the processes for environmental protection) how the principle of unit operations in combination with process integration works. Consider the very often used process for thermal disposal of polluted air. This process is used in a wide range of industries where one of the waste streams of production is a stream of polluted air. Typical users of such a process include car paint shops, bike paint shops and other products paint shops, but also smoking processes from food production, or manufacturers of various engineering dyed or lacquered products, etc. The actual process of thermal disposal of polluted air is very simple due to its high efficiency (i.e. output gas as the product from the thermal disposal of polluted air no longer needs to be further cleaned and can be discharged into the atmosphere) and thus includes only two unit operations - thermal oxidation and heat exchange. The unit operation of thermal oxidation is mostly realized in an equipment called combustion chamber, where thermal oxidation of polluted air occurs at the prescribed high temperature and resident time (depending on the specific polluting component in the air). The achievement of the required temperature is typically ensured by combustion of natural gas (as fuel) and combustion air in the burner. The thermal energy contained in the flue gas leaving the combustion chamber is then utilized by the second unit operation ("heat exchange") for a suitable purpose. Unit operation "heat exchange" is typically realized in (one or more) equipment called heat exchanger. The heat exchangers may be of different types and designs, depending on the purpose of utilizing the waste heat of the flue gas, as presented by Stehlík (2016). The initial configuration of the process thus assembled by unit operations is shown in Fig. 1a.

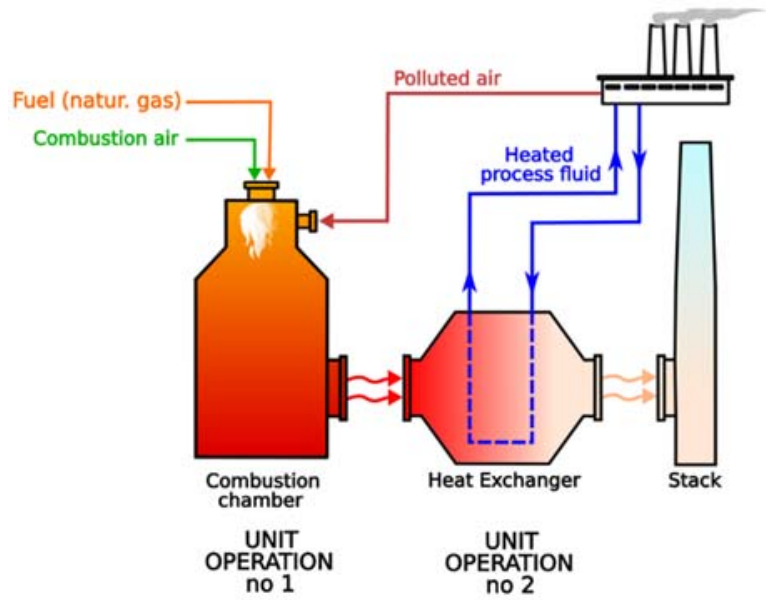

a) Initial process arrangement via unit operations.

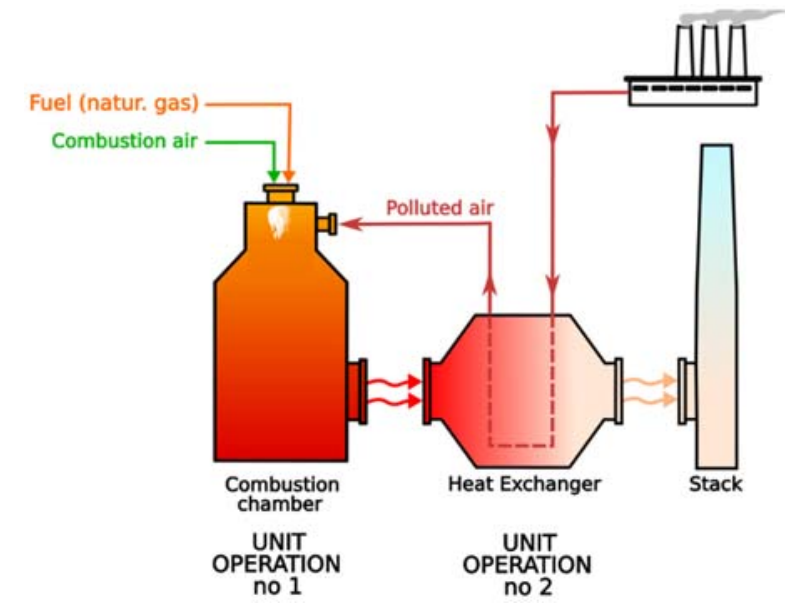

b) Final integrated process arrangement.

Fig. 1: Initial and integrated arrangement of process for thermal treatment of polluted air.

By subsequently applying the process integration method mentioned above, which utilizes specific manipulations of the system of cold and hot streams present in the process in connection with technicaleconomic trade-offs, an optimum integrated process design for the thermal disposal of the polluted air can be obtained, as shown in Fig. 1b. It can be seen from Fig. 1b that employing of process integration approach allows to find a process arrangement characterized by minimum investment and operating costs. Minimization of fuel (natural gas) consumption (and thus process operating costs) is achieved in that the waste heat of the flue gases leaving the combustion chamber is optimally utilized for preheating the polluted air in the heat exchanger.

In the context of global change and the need for maximum efficient, energy saving and environmentally friendly designs of production processes, efforts are now being made to upgrade the process integration approach to a higher level, and thus so-called "modern integrated equipment" (MIE) are being created. The aim of the paper is to introduce innovative philosophy of industrial MIE concepts and, above all, to highlight a several challenges that MIE proposals bring together with identification of relevant key areas of engineering mechanics that can help solve them and to which a special attention will be also paid. 


\section{Methods}

The one MIE can be generally characterized as an optimally designed multifunctional compact apparatus with high efficiency suitable aggregating a several unit operations into a single equipment (apparatus). The main motivation of MIE employing in the process is a reduction the process to the minimum number of spatially smaller equipment, and thus reduce both the investment and operating costs of the process. Moreover, a process consisting from a small number of MIE will work more efficiently and more environmentally friendly.

\subsection{The philosophy and example of MIE design}

The efficient design of any MIE should be based on the two basic assumptions: (a) arrangement of the process to which the MIE should be designed is a result of process integration method; (b) the MIE should be designed to suitable cover the two or more closest unit operations in the integrated process into a single equipment (apparatus).

Let us to demonstrate the MIE design philosophy via simple example of industrial process for thermal treatment of polluted air which was presented above in chapter Introduction. Remember, that our final process configuration after application of process integration approach consists of two "unit operations" respectively pieces of equipment: a combustion chamber and a heat exchanger for preheating polluted air (see Fig. 1b). Such a process, which has two pieces of equipment connected with piping, also involves thermal expansions systems, bulky refractory lining and a thick layer of insulation and thus occupies a relatively large space and a built-up area. Our aim is to design this process as MIE that will integrate both existing single unit operations into one equipment. Since the process meets both assumptions for an efficient MIE design, we can design it.

There are currently no specific rules for the design of the MIE, and it is up to the designer to decide which direction he will take when designing the MIE. In our case, we focused on eliminating heat losses to the environment from the combustion chamber. For this purpose, we have "wrapped" the combustion chamber with the polluted air preheater. This aim results in the specific developed MIE in which the polluted air is preheated in a special heat exchanger consisting of concentric sheets (cylinders). A cylindrical combustion chamber, which is the core of the process for the thermal treatment of gas wastes, is placed inside the heat exchanger. This MIE, which principal scheme is presented in Fig. 2, was also successfully industrially implemented.

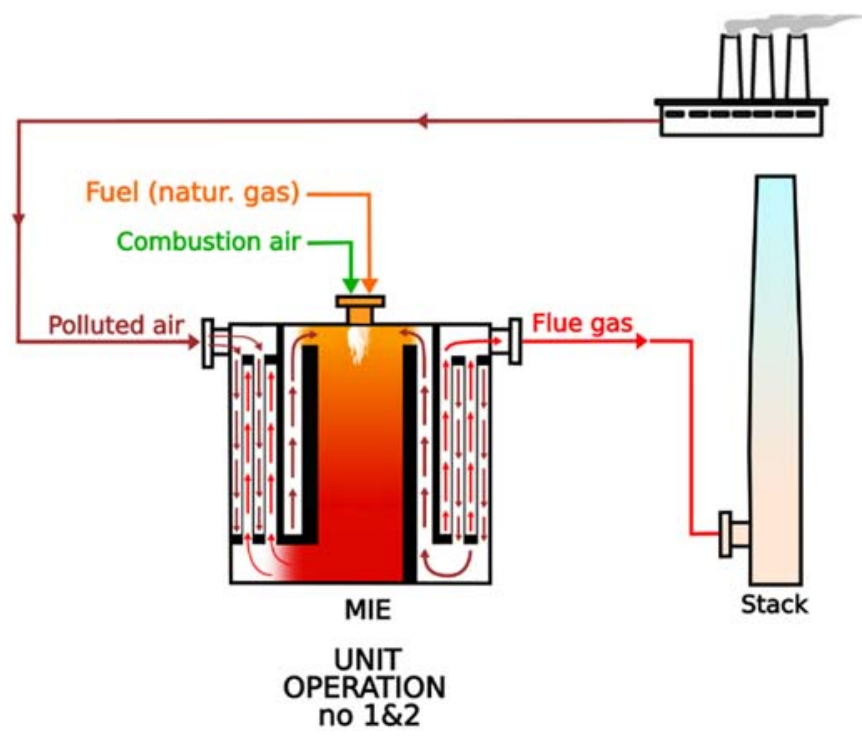

Fig. 2: Design scheme of MIE for thermal treatment of polluted air.

The presented MIE, which can be characterized as a integration of the combustion chamber and the polluted air pre-heater into a highly compact equipment, has several advantages: (i) the MIE fully integrates whole process into one multifunctional equipment; (ii) the MIE has relatively small dimensions due to its compactness; (iii) heat losses from the combustion chamber are utilized for preheating the polluted air; and (iv) the fuel (natural gas) flow rate is minimized as are heat losses to the ambient air. 


\subsection{Key areas of Engineering Mechanics in MIE design}

However, the current MIE design process is not smooth or simple. Process engineers who design MIE typically face several engineering mechanics challenges that are not yet satisfactorily addressed and hinder MIE being widely deployed and implemented in production processes. The essential challenges that we will now present can also be demonstrated in the above-mentioned case of MIE. Based on our experience with MIE development, we have identified the following key areas of engineering mechanics that we believe are crucial to MIE design:

The lack of reliable computational methods for designing realizable multifunctional equipment: As an example of this challenge it can be mentioned, for example, a necessity to develop a reliable computational method for thermal-hydraulic design of heat exchanger's part of the above-mentioned MIE, where the countercurrent helical flow of fluids (i.e. preheating polluted air and cooled down flue gas) occurs in its channels realized by concentric sheets (cylinders).

The problems with nonuniform fluids flow distribution, heat transfer, mechanical stress and tightness of parts within the integrated equipment: As example of these challenges can be again consider a heat exchanger's part of the above-mentioned MIE, where these problems occurred in the prototype and had to be addressed. The very time and cost consuming CDF and FEM analyses must be currently used to solve these problems. The development of alternative modeling methods that are comparably accurate but significantly less time and cost-intensive seems to be an appropriate way to address this challenge.

The need for wider international sharing of experience: This need is generally limited in the commercial sphere by an understandable effort to protect individual know-how. A successful example of addressing this challenge is, for example, the cooperation of Czech, German and Togolese universities in developing an integrated process and equipment for the needs of specific vegetable waste-to-energy processes substantial for developing countries and contributing to circular economy strategy, as presented by Azouma et al. (2019).

\section{Conclusions}

The paper presents in a concentrated way a process engineering specialization for experts in engineering mechanics. It explains the fundamental principles of the so-called unit operations on which a modern process engineering is based and demonstrates the extraordinary importance of the so-called process integration allowing to design a process characterized by minimum investment and operating costs. However, the key message of the paper is a presentation of the current trend in process engineering, which is the deployment of so-called modern integrated equipment (MIE) to further increase the quality of the design and operation of the process to an even higher level as a result of the current global changes that trigger these requirements. The paper finally discusses the most important challenges of engineering mechanics, which are currently crucial for the design of high-quality MIE and which in the future will significantly affect the real rate of expansion and use of MIE in production processes.

\section{Acknowledgement}

This research was supported by the EU project Strategic Partnership for Environmental Technologies and Energy Production, funded as project No. CZ.02.1.01/0.0/0.0/16_026/0008413 by Czech Republic Operational Programme Research, Development and Education, Priority Axis 1: Strengthening capacity for high-quality research.

\section{References}

Klemeš J. J., Varbanov P. S., Wan Alwi S. R., Manan Z.A. (2018) Process Integration and Intensification, Saving Energy, Water and Resources, $2^{\text {nd }}$ ed. De Gruyter.

Stehlík P. (2016) Up-to-Date Waste-to-Energy Approach. From Idea to Industrial Application, $1^{\text {st }}$ ed. SpringerBriefs in Applied Sciences and Technology, Springer International Publishing.

Azouma Y.O., Drigalski L., Jegla Z., Reppich M., Turek V. Weiß M. (2019) Indirect Convective Solar Drying Process of Pineapples as Part of Circular Economy Strategy, Energies, 12, 2841, pp. 1-12. 